HRJ v.2 n.12 (2021)

Recebido: 04/07/2021

Aceito: 24/08/2021

\title{
Planificação matusiana para monitoramento da hipertensão arterial sistêmica: a experiência de uma Unidade Básica de Saúde do Distrito Federal
}

\author{
Raynner Augusto Moreira Parente ${ }^{1}$ \\ Pedro Alexandre Barreto Coelho ${ }^{2}$
}

\footnotetext{
${ }^{1}$ Residente do Programa de Residência em Medicina de Família e Comunidade da SES-DF

${ }^{2}$ Preceptor do Programa de Residência em Medicina de Família e Comunidade da SES-DF
}

\begin{abstract}
RESUMO
A Atenção Primária é a "porta de entrada" aos serviços de saúde, contando com uma rede descentralizada de grande capilaridade. As Unidades Básicas de Saúde são os locais onde são desempenhadas as ações de alta complexidade e baixa densidade, mas de grande variedade segundo as necessidades da população atendida. Diante da diversidade de demandas atendidas nas unidades básicas, é necessário desenhar um planejamento estratégico como forma de organizar e melhorar a eficiência e qualidade do serviço prestado. Para tal foi utilizado o método Altair de planificação popular (MAPP), proposto por Carlos Matus, como arcabouço do planejamento estratégico situacional. Para encontrar as dez principais doenças da população Unidade Básica de Saúde número 15 de Ceilândia-DF, foram utilizados dados de consultas individuais do sistema SIAB/e-SUS, no intervalo de 01 de janeiro de 2018 até 30 de novembro de 2018. Após uma classificação de prioridade, utilizando os critérios de transcendência, urgência e capacidade, a Hipertensão Arterial Sistêmica foi selecionada para análise, com o objetivo final de construir um plano de ação para o seu controle rígido na unidade. O plano de ações alfa específicas incluiu, dentre outros, o aumento no número de consultas, aumento do número de matriciamento, qualificação de toda a equipe da Estratégia de Saúde da Família (ESF) e visitas dos agentes comunitário de saúde (ACS) com aparelhos para aferição de PA. A maioria das ações é relacionada principalmente à dinâmica de equipe que inicialmente encontrava lacunas em seus protocolos. Com a efetiva implementação do plano de ação será possível melhorar os determinantes de saúde dessa população e diminuir a incidência e comorbidades relacionadas à hipertensão arterial sistêmica (HAS).
\end{abstract}

Palavras-chave: Hipertensão Arterial Sistêmica, Atenção Primária à Saúde, Planejamento Estratégico, Saúde da Família

\section{Matusian planning for monitoring systemic arterial hypertension: the experience of a basic health unit in the Federal District}

Primary care is the "gateway" to health services, with a decentralized network of great capillarity. In the Basic Health Units actions of high complexity but of great variety are performed, according to the needs of the assisted population. Given the diversity of demands met in the basic units, it is necessary to design strategic planning as a way to organize and improve the efficiency and quality of the service provided. For this, the 
Altair method of popular planning, proposed by Carlos Matus, was used as a framework for situational strategic planning. To find the top ten diseases of the Basic Health Unit number 15 of Ceilândia-DF population, data from individual consultations of the SIAB / e-SUS system were used, from January 1, 2018 to November 30, 2018. After a Priority classification, using the criteria of transcendence, urgency and capacity, Systemic Arterial Hypertension was selected for analysis, with the ultimate goal of building an action plan for its strict control in the unit. The specific alpha action plan included, among others, the increase in the number of consultations, the increase in the number of matrices, the qualification of the whole team and visits with pressure gauges. Most actions are mainly related to the team dynamics that initially failed. With the effective implementation of the action plan it will be possible to improve the health determinants of this population and reduce the incidence and comorbidities related to hypertension.

Key words: Hypertension, Primary Health Care, Strategic Planning, Family Health

\section{INTRODUÇÃO}

A Atenção Primária a Saúde (APS) é um amplo segmento de saúde em que são abordadas diversas situações, sejam biológicas, sociais, mentais e culturais, sendo assim uma grande oportunidade para estudar populações específicas. O Relatório Dawson do Ministério da Saúde do Reino Unido em 1920 descreve a Unidade Básica de Saúde como principal organizador da APS. O profissional generalista possui importante papel de responsabilidade nessa cadeia, gerando as ações para organização das diferentes equipes e profissionais ${ }^{1,2}$

Além do papel organizador, cerca de $85 \%$ de todas as demandas são solucionadas na APS, favorecendo que esse ambiente seja de extrema riqueza para estudo. ${ }^{3} \mathrm{Na}$ planificação da atenção à saúde, a atenção básica é considerada um laboratório de inovação ${ }^{2}$

Esse estudo faz parte de um projeto de conclusão de curso de uma especialização em saúde da família e foi tomada como base a utilização do planejamento estratégico para gerar uma análise minuciosa de um determinado agravo selecionado. $\mathrm{O}$ planejamento foi utilizado para investigar, organizar as ações e compreender o problema de saúde da Unidade Básica de Saúde (UBS) número 15 de Ceilândia-DF. 
O estudo está inserido em um dos grandes deveres da Atenção Básica (AB) que é, segundo a última Política Nacional da Atenção Básica (PNAB), diagnóstico em saúde. Essenciais para o diagnóstico são as práticas de cuidado integrado, multiprofissional e com gestão qualificada, dirigidas à população do território de atuação da unidade básica ${ }^{4}$

A técnica utilizada foi o planejamento estratégico situacional que serve para gerenciar o processo de trabalho, com o objetivo de sempre melhorar a assistência. Dessa forma, facilita o encontro de objetivos e desenvolvimento de ações interessantes a serem aplicadas na comunidade. Através desse monitoramento, realizado primeiramente de maneira empírica e posteriormente de maneira cartesiana, foram selecionados temas de extrema prevalência e importância na unidade, chegando ao alvo principal desse estudo.

O planejamento estratégico é uma metodologia de grande importância que pode ajudar e muito o fortalecimento da estratégia de saúde da família e, portanto, da atenção básica à saúde. Favorecendo o foco nas doenças mais frequentes para a população específica da unidade, torna as ações de saúde mais efetivas. Assim como realizado no estudo feito na Bahia onde esse método foi utilizado por uma turma de faculdade para sistematizar uma ação em relação à promoção à saúde de idosos no município gerando bons resultados 5

Assim como visto anteriormente, há necessidade de foco específico para uma população. Segundo Reichert et al. para que seja possível a orientação familiar é necessário não somente responder a um problema e sim observar o indivíduo em sua integralidade. Para tanto, é necessário analisar o indivíduo no seu ambiente social, no seu contexto familiar e na sua exposição a ameaças da saúde. Isso muda um pouco quando o objetivo do planejamento estratégico é para uma comunidade como um todo. 
Para isso, a orientação comunitária tem que considerar se as necessidades de saúde das pessoas estão relacionadas, mostrando mais uma vez a necessidade do reconhecimento da realidade social dos pacientes pela equipe ${ }^{6 .}$

Corroborando essa ideia, um relato de experiência feito por um residente multiprofissional da região Sul do Brasil, mostrou que a planificação da Atenção Básica a Saúde (APS) favorece uma mobilização das equipes para a qualificação do trabalho realizado, além de mostrar a necessidade de um apoio multidisciplinar. O mesmo artigo também exemplifica, porém os diversos entraves na composição de uma planificação, principalmente a resistência a mudanças no processo de trabalho pelo os servidores e a falta de compromisso de todos, gestores, profissionais e população ${ }^{7}$.

Apesar dessa dificuldade, presente em qualquer processo de mudança de hábitos, a planificação traz diversas melhorias em geral, como mostra Costa, 2019, no seu trabalho. Costa mostrou que, após uma planificação realizada em um município do interior de Goiás, houve melhora nas condições de trabalho e otimização dos serviços para a população. Dentre as ações planificadas, foram modificadas as agendas, a organização das triagens e os prontuários para melhor atender aquela população em particular $^{8}$

Logo, para esse estudo foi utilizado o método Altair de planificação popular (MAPP), proposto por Carlos Matus, com arcabouço do planejamento estratégico situacional, seguindo quatro momentos: explicativo, normativo, estratégico e táticooperacional. Espera-se que através da utilização da planificação popular de Matus, seja possível gerar um plano de ação para comunidade com objetivo de melhora situacional ${ }^{9}$. Com a grande expansão da estratégia de saúde da família, como principal programa de saúde do Ministério da Saúde, e uma política de escolha a ser empregada em todo o país, houve a necessidade de transformações. Principalmente houve modificação no 
foco do atendimento ao usuário, substituindo o atendimento médico-centrado pelo centrado no paciente como um todo. Também foram atualizadas as definições de saúde, deixando de ser uma mera ausência de doenças, para uma visão holística do indivíduo. Com isso houve uma maior ânsia por novos estudos e um conhecimento mais especializado das populações a serem tratadas 9 .

O planejamento estratégico abrange a definição das seguintes etapas: identificar a situação dessa população com possíveis explicações situacionais; definir os objetivos a serem alcançados; construir metas para alcançar os objetivos; avaliar a viabilidade desses planos; e definir ações para que se alcance o objetivo. Foram levantadas as dez mais importantes enfermidades e dessas, foi selecionada a hipertensão arterial sistêmica, que faz parte das duas doenças mais diagnosticadas na unidade e uma das responsáveis pela maior causa de mortes no mundo, que são as doenças cardiovasculares ${ }^{10}$.

Hipertensão Arterial Sistêmica (HAS) é uma das grandes causadores de mortes no Brasil e no mundo, pois está dentro das doenças cardiovasculares e esse é a maior causa de mortalidade mundial, segunda a Organização Mundial da Saúde em $2005^{11 .}$ Os dados mais recentes retirados do Sistema de Informações de Mortalidade (SIM), do Ministério da Saúde, são ainda muito preocupantes. Ocorreram cerca de 34 óbitos por hora, 829 óbitos por dia e mais de 302 mil óbitos no ano de 2017 por doenças cardiovasculares no Brasil (infarto, hipertensão, AVC e outras enfermidades). O maior fator de risco para todas é a hipertensão arterial $^{12}$.

Ainda segundo dados do Sistema de Vigilância de Fatores de Risco e Proteção para Doenças Crônicas por Inquérito Telefônico (Vigitel 2018), em 2018, 24,7\% da população que vive nas capitais brasileiras afirmaram ter diagnóstico de hipertensão, sendo mais prevalente nos idosos (60,9\% possuem HAS). A pesquisa foi realizada por 
telefone com 52.395 pessoas maiores de 18 anos, entre fevereiro e dezembro do ano passado de $2018^{13}$

HAS é um agravo também muito estudado no âmbito de atenção básica, como feito por Rolim, 2019. Seu trabalho teve como objetivo elaborar um melhor diagnóstico e acompanhamento de pacientes com hipertensão arterial e diabetes mellitus na população do Itapoã-DF, através do conhecimento acerca da população e suas dificuldades para seguir o tratamento adequado ${ }^{14}$

Esses dados mostram a real dimensão da hipertensão arterial e do seu potencial número de óbitos ao longo dos anos. Por outro lado, várias medidas de prevenção podem ser adotadas na atenção primária para controlar a hipertensão e evitar desfechos negativos, tornando o presente estudo muito importante para a população assistida pela Equipe 21 da UBS 15 de Ceilândia-DF.

Por fim, para o levantamento situacional realizado nesse estudo foram utilizadas diversas ferramentas epidemiológicas bastantes descritas em estudo de planificação. Além dessas tecnologias, foram consideradas as condições de saúde da população, compreendendo a evolução do quadro epidemiológico e seus fatores de risco, além da vulnerabilidade aos agravos.

A Planificação da Atenção Primária à Saúde é uma prática apoiada e estimulada pelo Conselho Nacional de Secretários de Saúde (CONASS), com o objetivo de frisar o papel da Atenção Primária Saúde como ordenadora da rede. Logo, o método de planificação é muito utilizado corroborando sua utilização nesse estudo, que será de grande importância para a população ${ }^{8}$.

Com a elaboração final de uma ação, será possível uma mudança real dos determinantes de saúde dessa população e diminuir com isso a incidência dessa doença. Perspectiva essa endossada por trabalhos anteriores já realizados em outras unidades da 
federação e com resultados positivos. Como Costa (2019) na região de Goiás, Rolim (2019) em Itapoã-DF entre outros ${ }^{8,14}$ Exemplos exitosos como os citados mostram a grande necessidade de realização de estudos mais específicos para cada população.

Rolim, et al. criaram uma tabela que incluía informações de cada paciente em relação a consultas realizadas, fatores de risco para hipertensão e diabetes, escala de CoelhoSavassi, se era hipertenso ou diabético. Após a análise dos dados, foi observado que cerca de apenas 60,4\%, em 2016, foram atendidos, mostrando uma dificuldade no seguimento desses pacientes. As principais causas na falha de acompanhamento dos pacientes foram as longas jornadas de trabalho, sobrecarga de agenda e demanda reprimida. Foi então traçado um objetivo de cobertura de $100 \%$ dos atendimentos para os pacientes com hipertensão ou diabetes ${ }^{14}$ O estudo mostrou a importância do conhecimento da população e justificou a necessidade do presente estudo, considerando a realidade de uma UBS da região administrativa mais populosa do Distrito Federal.

A determinação de ações específicas construídas por meio da planificação facilitam o controle de uma doença na população alvo com maior acurácia e especificidade. Para avaliar tais mudanças na comunidade é possível avaliar os indicadores de saúde já utilizados.

A HAS é uma doença de alta prevalência na população brasileira que causa doenças cardiovasculares, renais e lesões retinianas, trazendo prejuízos econômicos e sociais para a população. Seu diagnóstico precoce e controle diminuem o risco do desenvolvimento de comorbidades e de óbito ${ }^{17}$. Na atenção básica, é possível detectar sinais e sintomas iniciais de HAS e tomar medidas de prevenção primária evitando aumento de sua prevalência.

Para chegar ao assunto a ser abordado nesse estudo, que fosse de importância específica para a população adscrita da ESF 21 da UBS 15 de Ceilândia-DF, foi 
utilizado Método Altair de Planificação Popular (MAPP), de Carlos Matus (MATUS, 1993). Foi fundamentado nos princípios do Planejamento Estratégico Situacional (PES), com objetivo de ser aplicado em nível local. Esse método já foi testado em toda a América Latina ${ }^{15,16}$. Depois de aplicada a planificação e encontrada a hipertensão arterial sistêmica como principal problema da comunidade, agora é necessário entender sobre essa doença, método de diagnóstico e sua importância no âmbito global e local.

As doenças crônicas não transmissíveis (DCNT) são responsáveis, segundo a Organização Mundial da Saúde (2008-2013), por cerca de 60\% das causas de mortes em todo mundo. Há uma expectativa de piora desse cenário com um aumento de $17 \%$ na mortalidade causada pelas DCNT. Um dos principais agravos relacionados a esses números são as doenças cardiovasculares, impactando em cerca de $30 \%$ de todas as mortes no mundo ${ }^{17}$ Logo, a hipertensão é uma das maiores causadoras de morte na população global. Se esse agravo não é controlado também pode gerar uma série de complicações, como doenças cardiovasculares e renais.

Para entender a magnitude dessa doença no âmbito nacional, $21 \%$ dos indivíduos com 18 anos ou mais (31,3 milhões) apresentaram diagnóstico prévio de HAS e 6,2\% (9,1 milhões) de Diabetes Mellitus, segundo dados da Pesquisa Nacional de Saúde de $2013^{17}$. No Brasil, as DCNT possuem uma representatividade até maior que no resto do mundo, sendo responsáveis por $72 \%$ das causas de mortes, com destaque para doenças do aparelho circulatório (31,3\%), câncer (16,3\%), diabetes (5,2\%) e doença respiratória crônica $(5,8 \%)^{18}$.

A prevalência só está piorando. Segundo dados do Sistema de Vigilância de Fatores de Risco e Proteção para Doenças Crônicas por Inquérito Telefônico (Vigitel 2018), em 2018, 24,7\% da população que vive nas capitais brasileiras afirmaram ter diagnóstico de hipertensão, sendo nos idosos ainda mais prevalente $(60,9 \%)$. No mesmo 
levantamento, as capitais com maior prevalência são Rio de Janeiro (31,2\%), Maceió (27,1\%), João Pessoa (26,6\%), Belo Horizonte (26,5\%), Recife $(26,5 \%)$ e Campo Grande (26,0\%). E as com menores índices são São Luís (15,9\%), Porto Velho (18,0\%), Palmas e Boa Vista $(18,6 \%)^{13 .}$

Os dados mais recentes do Sistema de Informações de Mortalidade (SIM), do Ministério da Saúde, são ainda mais alarmantes. Em 2017, o Brasil registrou 141.878 mortes devido à hipertensão ou a causas atribuíveis a ela. Hipertensão é também o maior fator de risco para doenças cardiovasculares (infarto, hipertensão, AVC e outras enfermidades) que causaram em 2017, cerca de 34 mortes por hora, 829 óbitos por dia e mais de 302 mil óbitos no Brasil ${ }^{12 .}$ Logo, a HAS é associada direta ou indiretamente a mais de 440 mil mortes por ano.

Diante desse panorama, a HAS se torna um importante alvo nas ações programáticas nos serviços de atenção primária à saúde (APS), devido à sua alta prevalência, à sua grande participação nas causas de morbimortalidade no país e por ser possível sua prevenção nesse nível de atenção. Apesar disso, segundo o estudo SERVIDIAH o controle da HAS continua muito insatisfatório, pois com uma amostra representativa de hipertensos, observou-se que menos metade tinha pressão arterial controlada $^{19}$

Na Pesquisa Nacional de Saúde infelizmente foi possível observar um quadro alarmante em todo o país, devido à elevada carga de DCNT e de incapacidades. E isso deve elevar-se devido ao envelhecimento da população. Desta forma, o Ministério da Saúde iniciou a expansão da cobertura e melhoria da assistência na atenção básica ${ }^{18}$. Dados ainda piores relacionados ao controle da hipertensão foram observados na Índia, onde menos de $15 \%$ dos casos tem algum controle. ${ }^{20}$ Por outro lado, na Itália estudo feito com médicos na atenção básica conseguiu ter um controle bem sucedido em $82 \%$ 
dos pacientes que estavam em acompanhamento na unidade. $\mathrm{Na}$ mesma época, o controle geral da hipertensão era apenas de $35 \%$ na Itáliaa ${ }^{21 .}$ Isso mostra ser possível o controle de HAS com maior qualidade e maior cobertura de doentes na atenção primária à saúde.

A planificação gerando informações e diagnósticos situacionais de saúde apoiam a aplicação de políticas nacionais e regionais, e estratégias setoriais e intersetoriais para o combate as DCNT e seus fatores de risco. E como mostrado em três estudos a melhora da qualificação da atenção básica pode reduzir a carga dessas doenças nas classes de média e baixa renda $22,23,24$.

Apenas as três principais DCNT (diabetes, cardiopatias e AVC) levaram a uma perda na economia brasileira de US\$ 4,18 bilhões entre 2006 e $2015^{25}$ Já o custo anual para o tratamento, privado e público, apenas da HAS é de cerca de US\$ 671,6 milhões, um grande impacto financeiro ${ }^{26 .} \mathrm{O}$ controle pressórico e acompanhamento regular dos pacientes na atenção básica reduzem o custo do tratamento e o impacto econômico dessa doença e suas consequências.

As principais dificuldades no controle dessa moléstia são: a ausência de prescrição de medicamentos anti-hipertensivos, quando necessário; baixa adesão aos agentes anti-hipertensivos (definido como $<80 \%$ das doses tomadas conforme prescrito); ineficácia dos medicamentos prescritos $^{27}$. Isso mostra que o controle passa por diversos fatores com diferentes variáveis, sendo uma delas o diagnóstico da doença.

A sétima Diretriz Brasileira de Hipertensão Arterial de 2016 definiu hipertensão arterial sistêmica como uma condição clínica multifatorial caracterizada por elevação sustentada dos níveis pressóricos $\geq 140$ e/ou $90 \mathrm{mmHg}$, possuindo associação independente com morte súbita, acidente vascular encefálico (AVE), infarto agudo do miocárdio (IAM), insuficiência cardíaca (IC), doença arterial periférica (DAP) e doença 
renal crônica (DRC). Possui diversos fatores de riscos como idade (relação direta com aumento da idade), sexo e etnia (mulheres e raça negra), ingestão de sal (consumo excessivo), consumo de bebidas alcoólicas e sedentarismo ${ }^{28 .}$

Assim como a sétima diretriz Brasileira de hipertensão de 2016, a Nova diretriz de hipertensão da European Society of Hypertension (ESC) 2018 manteve o diagnóstico de hipertensão, quando pressão maior ou igual 140/90 mmHg. A ESC divide os pacientes conforme valores de pressão respeitando a tabela $1^{29}$.

Tabela 1 - Estágios da pressão segundo ESC

\begin{tabular}{|l|l|l|}
\hline ESC 2018 & PA sistólica $(\mathbf{m m H g})$ & PA diastólica $(\mathbf{m m H g})$ \\
\hline Ótimo & $<120$ & $<80$ \\
\hline Normal & $120-129$ & $80-84$ \\
\hline Normal-Alto & $130-139$ & $85-89$ \\
\hline Estágio 1 & $140-159$ & $90-99$ \\
\hline Estágio 2 & $160-179$ & $100-109$ \\
\hline Estágio 3 & $\geq 180$ & $\geq 110$ \\
\hline
\end{tabular}

Fonte: Diretriz de HAS da European Society of Hypertension 2018

Ambas as diretrizes divergem no valor para diagnóstico de hipertensão arterial sistêmica definido pela American College of Cardiology Foundation e pela American Heart Association (AHA) 2017. Seguindo sua tradição de vanguarda, a AHA adotou valores menores para diagnóstico, sendo hipertensão quando a pressão arterial sistólica $(\mathrm{PAS}) \geq 130 \mathrm{mmHg}$ e/ou pressão arterial diastólica $(\mathrm{PAD}) \geq 80 \mathrm{mmHg}$ (tabela 2$)^{30}$.

Tabela 2 - Classificação da pressão segundo AHA

\begin{tabular}{|l|l|l|l|}
\hline Classificação & PA sistólica $(\mathbf{m m H g})$ & & PA diastólica $(\mathbf{m m H g})$ \\
\hline Normal & $<120$ & E & $<80$ \\
\hline Elevada & $120-129$ & E & $<80$ \\
\hline Hipertensão estágio 1 & $130-139$ & e/ou & $80-89$ \\
\hline Hipertensão estágio 2 & $\geq 140$ & e/ou & $\geq 90$ \\
\hline
\end{tabular}


O diagnóstico é simples requerendo apenas um aparelho de aferir pressão, e é confirmado quando em duas consultas diferentes o paciente tem pressão maior que 140x90 mmHg ${ }^{28 .}$ Segundo a ESC, se o paciente estiver no estágio 3 ou possuir lesão do órgão-alvo já instalada, uma segunda consulta não é necessária, sendo imediatamente iniciado o tratamento. Quanto maior a PA basal, consultas mais frequentes, com menor intervalo, são necessárias ${ }^{29}$.

Além do diagnóstico, são necessários exames de rastreio para investigação de doenças associadas e ou doenças que causem hipertensão (hipertensão secundária). Os exames solicitados, em geral, são simples como dosagem sérica de potássio, glicemia, creatinina, lipidograma, função renal, ácido úrico, exame de urina EAS e eletrocardiograma ${ }^{25}$.

Já o tratamento dessa moléstia é relativamente de baixo custo, com uma grande oferta de várias classes de medicamentos com distribuição no país. O tratamento tem como alvo pressórico, segundo a diretriz brasileira, pressão menor que 140x90 mmHg para a população hipertensa geral e para paciente com risco cardiovascular alto, pressão alvo menor que $130 \times 80 \mathrm{mmHg}^{28}$. Segundo a nova diretriz da ESC se o paciente tolerar, evoluir para pressões $<130 / 80 \mathrm{mmHg}$, evitando PAS $<120 \mathrm{mmHg}$ em paciente de qualquer idade e PAS $<130 \mathrm{mmHg}$ em idosos com mais de $65 \operatorname{anos}^{29}$.

O objetivo geral deste artigo foi apresentar um plano de ação para o manejo da hipertensão arterial sistêmica na Estratégia de Saúda da Família do Distrito Federal.

\section{MÉTODOS}

Nesse estudo foi realizada uma construção de ação para futura intervenção apoiado no planejamento estratégico segundo o método de Carlos Matus, usando a 
planificação. Logo, o trabalho é um estudo qualitativo, aplicado, explicativo e de campo.

Os dados foram referentes à unidade de saúde 15 de Ceilândia. Essa localidade possui acesso ao sistema SIAB/e-SUS. Foi utilizado o sistema para análise situacional da população atendida pela equipe de saúde da família 21 .

Foram utilizados os dados colhidos pelo sistema na parte de consultas individuais realizadas pela equipe. Dentro desse critério, ainda foram utilizados dois filtros, com objetivo de chegar mais facilmente ao diagnóstico situacional. Os limitadores utilizados foram diagnóstico pelo CID 10 e diagnóstico "outros".

Primeiramente planejou-se utilizar os dados relativos há um ano, ou seja, desde 01 de novembro de 2017 a 01 de novembro de 2018. No entanto, devido a uma limitação do sistema não foi possível utilizar essa janela temporal, sendo estipulado o intervalo de 01 de janeiro de 2018 até 30 de novembro de 2018.

Foi gerada uma tabela com todos os agravos diagnosticados da unidade nesse período e posteriormente foi colocado em ordem decrescente de número de diagnóstico por moléstia. Chegaram-se então nas dez principais doenças dessa população.

Após encontrar as patologias mais frequentes, foi realizada uma classificação de prioridade. Para tal foram utilizados três critérios sendo eles: transcendência, urgência e capacidade. A definição usada de transcendência foi a capacidade de afetar diversos seguimentos da rede, já para urgência foram as possíveis consequências geradas pela não resolução do problema de imediato e capacidade seria a possibilidade de colocar os planos de melhora em prática. Após a aplicação dos critérios foram selecionados apenas os três primeiros agravos.

Posteriormente, foram elencadas as principais causas e consequências para cada uma das três doenças mais prevalentes. Dessas, somente uma foi selecionada, com o 
objetivo de aprofundar no estudo sobre o assunto, gerando seus principais determinantes sociais, econômicos, culturais, comportamentais, relacionados ao trabalho da UBS e relacionados ao acesso e qualidade do SUS.

Já para fundamentação teórica foram selecionados artigos dos bancos de dados: Medline, Scielo e Pubmed.

\section{RESULTADOS E DISCUSSÃO}

Foram gerados, a partir da fonte de informação selecionada, mais de 2820 diagnósticos realizados na unidade no intervalo de 11 meses. Isso mostra uma situação diversificada de diagnósticos. Desses, mais de 31 doenças ou agravos foram diagnosticados em mais de 10 pessoas, 17 diagnósticos em mais de 20 pessoas e apenas 11 diagnósticos foram feitos em mais de 30 indivíduos. Os dez agravos mais encontrados na unidade em ordem decrescente de prevalência estão descritos no quadro 1. Em seguida, foram descritos cada um dos agravos, para melhor conhecimento de cada um (Quadro 2).

Utilizou-se a matriz de prioridade para selecionar os três diagnósticos mais realizados. Para tal necessidade, foram selecionados três critérios exemplificados anteriormente: transcendência, urgência e capacidade. A escolha dos critérios seguiu fatores primordiais de um possível sucesso na aplicação da ação sobre a doença selecionada, pois tinha que ser algo prevalente, com certa importância na área da saúde, com necessidade de intervenção não muito emergencial e que fosse possível ser aplicado. 
Quadro 1 - Agravos mais encontrados na unidade em ordem decrescente de prevalência

\begin{tabular}{|l|l|}
\hline \multicolumn{2}{|l|}{ Agravo } \\
\hline 1 & Hipertensão essencial \\
\hline 2 & Diabetes Mellitus \\
\hline 3 & Diarreia \\
\hline 4 & Hipercolesterolemia pura \\
\hline 5 & Doença de refluxo gastroesofágico \\
\hline 6 & Dor lombar baixa \\
\hline 7 & Rinite \\
\hline 8 & Nasofaringite aguda \\
\hline 9 & Enxaqueca \\
\hline 10 & Gripe \\
\hline
\end{tabular}

Fonte: Elaboração própria de acordo com dados coletados

Quadro 2 - Descritores dos dez agravos mais prevalentes na UBS 15 de Ceilândia $\mathrm{DF}$

\begin{tabular}{|l|l|l|}
\hline \multicolumn{2}{|l|}{ Agravo } & $\begin{array}{l}\text { Descritores (quantidade de } \\
\text { pacientes diagnosticados em 2018) }\end{array}$ \\
\hline 1 & Hipertensão essencial & 252 \\
\hline 2 & Diabetes Mellitus & 75 \\
\hline 3 & Diarreia & 38 \\
\hline 4 & Hipercolesterolemia pura & 36 \\
\hline 5 & $\begin{array}{l}\text { Doença de refluxo } \\
\text { gastroesofágico }\end{array}$ & 35 \\
\hline 6 & Dor lombar baixa & 34 \\
\hline 7 & Rinite & 32 \\
\hline 8 & Nasofaringite aguda & 32 \\
\hline 9 & Enxaqueca & 31 \\
\hline 10 & Gripe & 30 \\
\hline
\end{tabular}

Fonte: Elaboração própria de acordo com dados coletados

Após detecção dos principais agravos, as doenças foram classificadas de acordo com os critérios transcendência, urgência e capacidade. Para cada critério foi dado um valor (em uma escala de até 10) e posteriormente os valores foram multiplicados. Os três agravos com maiores dígitos foram selecionados (Quadro 3), sendo eles hipertensão essencial, Diabetes Mellitus e diarreia. 
Quadro 3 - Matriz TUC para seleção dos agravos encontrados

\begin{tabular}{|l|l|l|l|l|l|}
\hline \multirow{2}{*}{\begin{tabular}{l} 
Agravo \\
\cline { 2 - 5 }
\end{tabular}} & \multicolumn{2}{|l|}{$\begin{array}{l}\text { CRITÉRIOS (Valores de 1 a 10) } \\
\text { Transcendência }\end{array}$} & $\begin{array}{l}\text { Urgência } \\
(\mathrm{U})\end{array}$ & $\begin{array}{l}\text { Capacidade } \\
(\mathrm{C})\end{array}$ & TOTAL \\
\hline 1 & Hipertensão essencial & 10 & 6 & 8 & 480 \\
\hline 2 & Diabetes Mellitus & 10 & 6 & 8 & 480 \\
\hline 3 & Diarreia C $)$ & 7 & 8 & 392 \\
\hline 4 & $\begin{array}{l}\text { Doença de refluxo } \\
\text { gastroesofágico }\end{array}$ & 7 & 7 & 10 & 350 \\
\hline 5 & Enxaqueca & 7 & 7 & 8 & 336 \\
\hline 6 & Gripe & 6 & 6 & 9 & 324 \\
\hline 7 & Nasofaringite aguda & 5 & 6 & 9 & 270 \\
\hline 8 & Dor lombar baixa & 6 & 6 & 7 & 252 \\
\hline 9 & Hipercolesterolemia pura & 6 & 6 & 8 & 240 \\
\hline 10 & Rinite & 4 & 5 & 8 & 160 \\
\hline
\end{tabular}

Fonte: Elaboração própria de acordo com dados coletados

Com as três moléstias selecionadas segundo a prioridade, foi realizada uma

análise, encontrando suas principais causas e consequências (Quadro 4).

Quadro 4 - Matriz para análise dos problemas selecionados

\begin{tabular}{|c|c|c|}
\hline Problema a & \multicolumn{2}{|l|}{ Hipertensão essencial } \\
\hline Descritores & Causas & Consequências \\
\hline $\begin{array}{l}252 \text { pacientes } \\
\text { diagnosticados em } \\
2018\end{array}$ & $\begin{array}{l}\text { Hereditário } \\
\text { Má alimentação } \\
\text { Sedentarismo } \\
\text { Doenças prévias }\end{array}$ & $\begin{array}{l}\text { AVC } \\
\text { IAM } \\
\text { Doença renal crônica } \\
\text { Dissecção de aorta } \\
\text { Aneurisma } \\
\text { Morte }\end{array}$ \\
\hline Problema b & \multicolumn{2}{|l|}{ Diabetes Mellitus } \\
\hline Descritores & Causas & Consequências \\
\hline $\begin{array}{l}75 \text { pacientes } \\
\text { diagnosticados em } \\
2018\end{array}$ & $\begin{array}{l}\text { Hereditário } \\
\text { Má alimentação } \\
\text { Sedentarismo } \\
\text { Doenças prévias }\end{array}$ & $\begin{array}{l}\text { AVC } \\
\text { IAM } \\
\text { Doença renal crônica } \\
\text { Cegueira } \\
\text { Amputação de membros } \\
\text { Morte }\end{array}$ \\
\hline Problema c & \multicolumn{2}{|l|}{ Diarreia } \\
\hline Descritores & Causas & Consequências \\
\hline $\begin{array}{l}38 \quad \text { pacientes } \\
\text { diagnosticados em } \\
2018\end{array}$ & $\begin{array}{l}\text { Falta de higiene } \\
\text { Falta de saneamento básico } \\
\text { Falta de conhecimento }\end{array}$ & $\begin{array}{l}\text { Falta ao trabalho } \\
\text { Infecção grave } \\
\text { Morte }\end{array}$ \\
\hline
\end{tabular}

Fonte: Elaboração própria de acordo com dados coletados

De acordo com o Quadro 4, as duas primeiras moléstias possuem fatores causais e consequências muito semelhantes do ponto de vista médico. Já a terceira possui 
causas mais relacionadas à qualidade de vida do indivíduo e seu grau de instrução e as consequências mais relacionadas ao trabalho.

Após uma observação realizada na comunidade e através da vivência na comunidade, foi possível detectar uma grande população com hipertensão arterial sistêmica sem controle. Ainda foi possível observar que hipertensão e diabetes são os dois maiores fatores de risco para lesão renal crônica. Logo, como hipertensão é a doença mais diagnosticada na unidade e a maior causadora de morte por doenças cardiovasculares, foi selecionada então como problema a ser estudado.

Para melhor compreender os motivos para o manejo inadequado da hipertensão arterial sistêmica foi elaborado o quadro 5 com a rede de determinação causal do problema.

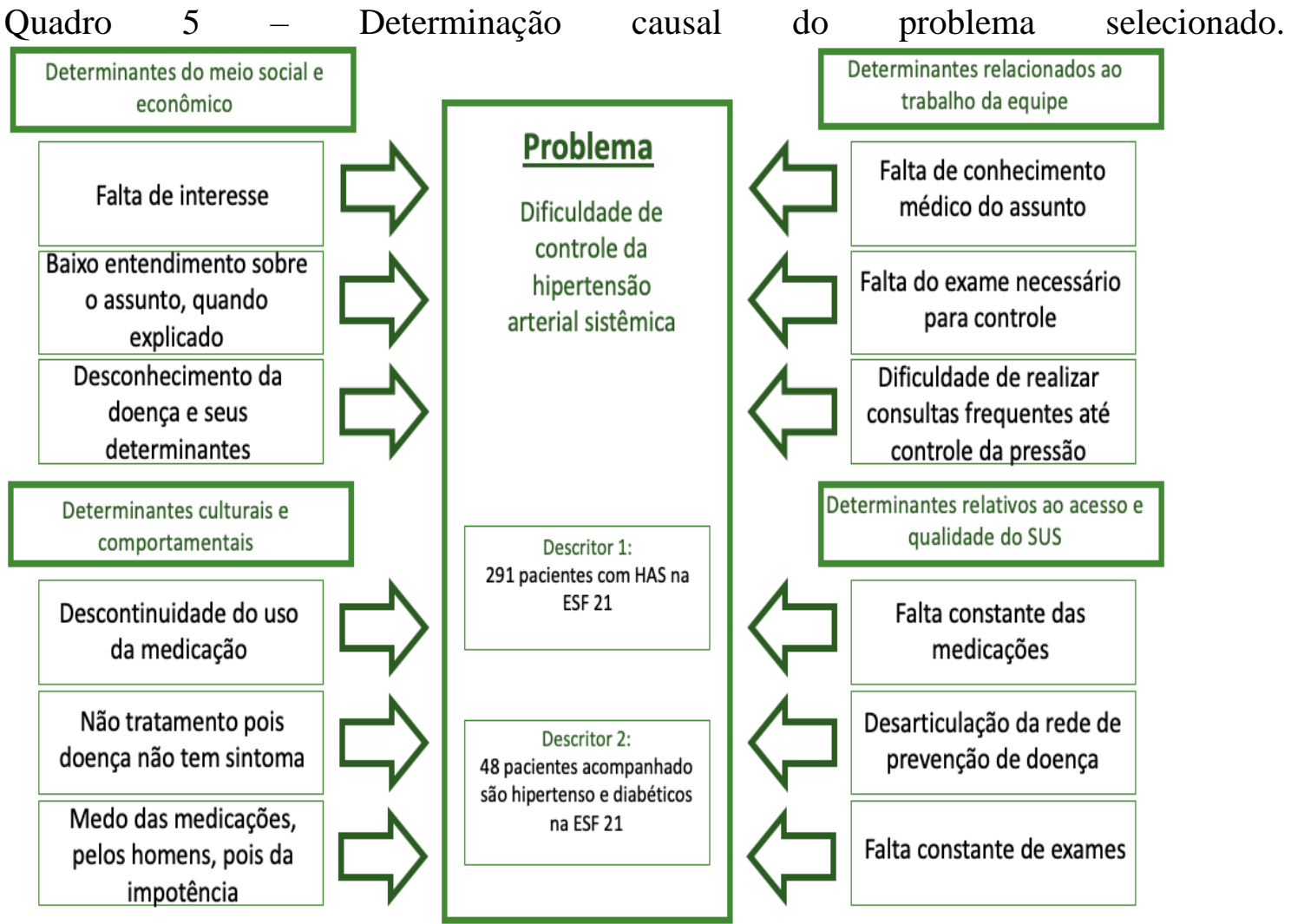

Fonte: Elaboração própria de acordo com dados coletados 
No geral, o principal problema encontrado para o controle da hipertensão é a falta de conhecimento, seja da equipe de saúde, seja dos pacientes. Ainda a dificuldade de realizar consultas frequentes até encontrar o tratamento adequado e o inconstante abastecimento de medicamentos são fatores que também tem grande influência negativa no controle do agravo.

Após levantar as principais justificativas ou determinantes para o difícil controle da pressão arterial nessa comunidade, foi produzido o plano de ação (quadro 6). Discutiu-se a necessidade de elaborar a planilha com todos os pacientes com o diagnóstico de diabetes e/ou hipertensão arterial e calcular a taxa de filtração glomerular de todos.

A ação final seria conhecer o total de indivíduos com a doença descontrolada e realizar marcação de consultas periódicas semanais e exames até chegar ao alvo da pressão arterial ideal. Já para os hipertensos controlados, orientar sobre a prevenção primária, secundária, promoção de saúde e marcar consultas semestrais com exames anuais.

Para melhor divisão das ações e avaliação do cumprimento de suas metas foi realizada a separação dos atos em alfa, beta e gama. Dentro dos objetivos tipo alfa ficaram as partes programáticas, que são o grande limitador ao cumprimento dos objetivos (quadro 7). Já os objetivos tipo beta são as ações necessárias e suficientes para o enfrentamento o problema (quadro 8) e por fim a tipo gama são as metas que vislumbram alcançar uma melhora após a missão já concluída, em busca sempre de novos avanços (quadro 9).

Os obstáculos da planificação foram discutidos e elencados em diversos estudos, sendo um deles o realizado por Dorneles, exemplificando a resistência frequentes dos servidores, principalmente os mais antigos, à mudança. A compreensão da importância 
da mudança e seu potencial de reduzir moléstia foi essencial para que essa barreira às mudanças fosse rompida ${ }^{7}$.

Esse obstáculo criado pelos servidores já foi amplamente estudado, e foi visto que mudanças trazem incertezas e turbulências no ambiente organizacional ${ }^{31,32 .}$ Isso por fim gera resistência, devido à necessidade de sair de uma situação conhecida para uma desconhecida $^{33 .}$ E esse mecanismo de resposta gera efeitos negativos na dedicação/comprometimento dos trabalhadores ${ }^{34}$ Já a justificativa final para o surgimento de resistência a mudança está bem explicada em cinco fatores: indecisão e inconclusão (conhecido é substituído pelo incerto); ameaça ao convívio social (ameaçam a integridade dos grupos de amizade); pressão do grupo; consistência organizacional (tarefas formalizadas e com necessidade de ser rigorosamente seguidas pelos funcionários); experiências prévias (experiências negativas anteriores pode criar atitudes de rejeição) ${ }^{35}$.

Quadro 6 - Plano de ações alfa, beta e gama

\section{Problema: Dificuldade de controle da hipertensão}

\begin{tabular}{|c|c|c|c|c|}
\hline $\begin{array}{l}\text { Ações } \\
\text { estratégicas }\end{array}$ & $\begin{array}{l}\text { Produtos } \\
\text { esperados }\end{array}$ & $\begin{array}{l}\text { Resultados } \\
\text { esperados }\end{array}$ & Responsável & $\begin{array}{l}\text { Início- } \\
\text { Fim }\end{array}$ \\
\hline $\begin{array}{l}\text { Alfa: Controle } \\
\text { mais eficaz da } \\
\text { HAS }\end{array}$ & $\begin{array}{l}1 \text { - Aumento no } \\
\text { número de } \\
\text { consultas para } \\
\text { os pacientes. } \\
2 \text { - Aumento do } \\
\text { número de } \\
\text { matriciamento, } \\
\text { capacitando o } \\
\text { profissional da } \\
\text { ESF } \\
3 \\
\text { Qualificação de } \\
\text { toda a equipe da } \\
\text { ESF, para } \\
\text { melhorar o } \\
\text { atendimento } \\
\text { desses } \\
\text { pacientes. } \\
4 \text { - Visitas dos }\end{array}$ & $\begin{array}{l}\text { 1 - Conhecer } \\
\text { exatamente a } \\
\text { epidemiologia da } \\
\text { HAS na } \\
\text { comunidade. } \\
2 \text { - Melhorar a } \\
\text { qualidade de vida } \\
\text { dos pacientes com } \\
\text { HAS. } \\
3 \text { - Prevenção } \\
\text { secundária para } \\
\text { evitar lesões } \\
\text { renais terminais, } \\
\text { infarto, AVE e } \\
\text { demais sequelas } \\
\text { graves; } \\
4 \text { - Aumento no } \\
\text { número de doentes } \\
\text { controlados para }\end{array}$ & $\begin{array}{l}\text { Médico e } \\
\text { enfermeiro } \\
\text { da unidade. }\end{array}$ & $\begin{array}{l}\text { Atividade } \\
\text { contínua }\end{array}$ \\
\hline
\end{tabular}




\begin{tabular}{|c|c|c|c|c|}
\hline & $\begin{array}{lr}\text { ACS } & \text { com } \\
\text { aparelhos } & \text { de } \\
\text { aferir a PA } & \end{array}$ & $\begin{array}{l}\text { HAS. } \\
5 \text { - Diminuição do } \\
\text { número de } \\
\text { encaminhamento } \\
\text { para } \\
\text { cardiologista. } \\
6 \text { - Diminuição da } \\
\text { progressão da } \\
\text { doença. }\end{array}$ & & \\
\hline $\begin{array}{l}\text { Beta: } \\
1 \text { - Melhorar a } \\
\text { disponibilidade } \\
\text { de medicação e } \\
\text { a facilidade de } \\
\text { realização de } \\
\text { exames de } \\
\text { controle; } \\
2 \\
\text { Confeccionar } \\
\text { uma tabela com } \\
\text { os dados dos } \\
\text { pacientes com } \\
\text { HAS e DM. }\end{array}$ & $\begin{array}{l}\text { 1 - } \text { Confecção } \\
\text { de } \\
\text { farmácia na } \\
\text { unidade. } \\
2 \text { - Coletar o } \\
\text { material para } \\
\text { exame na } \\
\text { unidade } \\
3 \text { - Planilha dos } \\
\text { pacientes com } \\
\text { HAS ou DM, } \\
\text { com a taxa de } \\
\text { função renal } \\
\text { calculada. }\end{array}$ & $\begin{array}{l}1 \text { - Aumentar a } \\
\text { adesão do paciente } \\
\text { ao tratamento. } \\
2 \text { - Conhecimento } \\
\text { de todos os } \\
\text { pacientes com } \\
\text { HAS ou DM. } \\
3 \text { - Aumentar o } \\
\text { diagnóstico de } \\
\text { hipertensão } \\
\text { secundária. } \\
4 \text { - Aumento da } \\
\text { vinculação do } \\
\text { paciente com a } \\
\text { unidade. }\end{array}$ & $\begin{array}{l}\text { Médico e } \\
\text { enfermeiro } \\
\text { da unidade. }\end{array}$ & $\begin{array}{l}\text { Atividade } \\
\text { contínua }\end{array}$ \\
\hline $\begin{array}{l}\text { Gama: } \\
1 \\
1 \\
\text { Diagnosticar } \\
\text { precocemente a } \\
\text { hipertensão } \\
\text { arterial } \\
\text { sistêmica nas } \\
\text { Unidades } \\
\text { básicas } \\
\text { saúde. }\end{array}$ & $\begin{array}{l}1 \text { - Lista de } \\
\text { pacientes com } \\
\text { HAS e/ou DM, } \\
\text { e dados como } \\
\text { obesidade, } \\
\text { circunferência } \\
\text { abdominal, } \\
\text { última consulta } \\
\text { etc. } \\
2 \text { - Solicitação } \\
\text { de exames } \\
\text { anuais do } \\
\text { publico com } \\
\text { fator de risco. } \\
3 \text { - Consulta } \\
\text { médica anual. } \\
4-\text { Palestra de } \\
\text { prevenção de } \\
\text { HAS para }\end{array}$ & 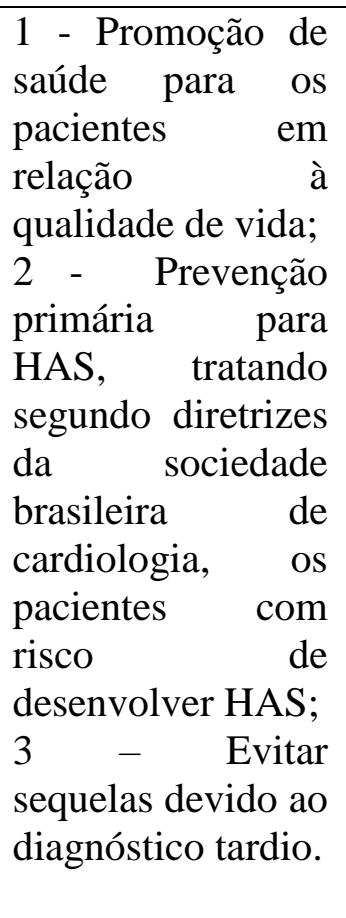 & $\begin{array}{l}\text { Toda equipe } \\
\text { da UBS }\end{array}$ & $\begin{array}{l}\text { Atividade } \\
\text { contínua }\end{array}$ \\
\hline
\end{tabular}




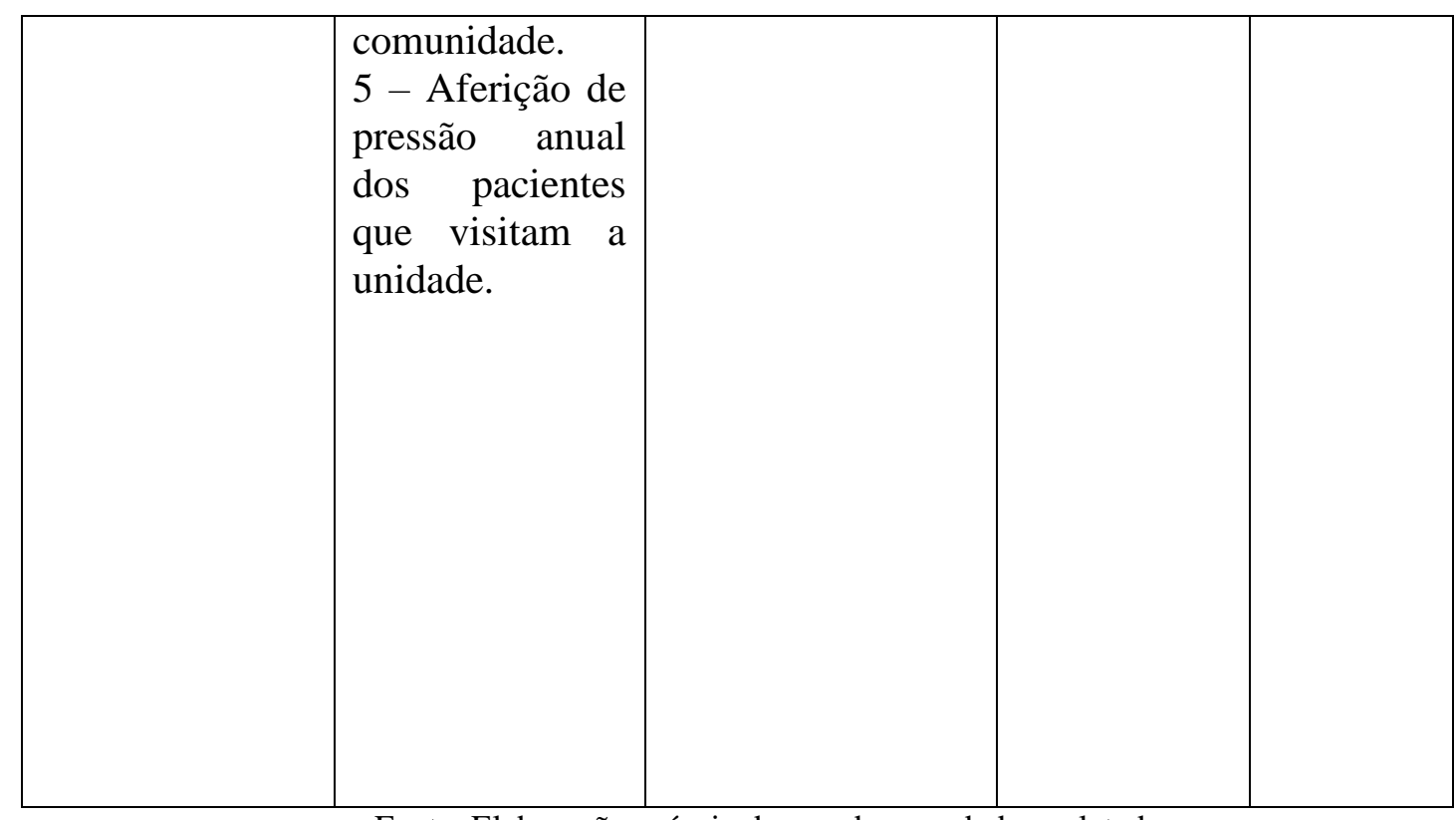

Fonte: Elaboração própria de acordo com dados coletados

Quadro 7 - Plano de ações alfa e suas metas

Problema: Dificuldade de controle da hipertensão

Objetivo: Controle mais eficaz da HAS

Meta de resultado: Evitar complicações e prevenção secundária

\begin{tabular}{|c|c|c|c|c|}
\hline $\begin{array}{l}\text { Ações do tipo } \\
\text { alfa }\end{array}$ & Meta de Produto & Responsável & Prazo & $\begin{array}{l}\text { Início- } \\
\text { Fim }\end{array}$ \\
\hline $\begin{array}{ll}\text { Aumento } & \text { no } \\
\text { número } & \text { de } \\
\text { consultas para } & \text { os } \\
\text { pacientes. } & \end{array}$ & $\begin{array}{ll}\text { Melhorar } & \text { a } \\
\text { qualidade de vida } \\
\text { dos pacientes com } \\
\text { HAS. }\end{array}$ & Dr. Raynner & $\begin{array}{l}\text { Atividade } \\
\text { contínua }\end{array}$ & 2019 \\
\hline $\begin{array}{lr}\text { Aumento } & \text { do } \\
\text { número } & \text { de } \\
\text { matriciamento, } & \\
\text { capacitando } & \text { o } \\
\text { profissional } & \text { da } \\
\text { ESF } & \end{array}$ & $\begin{array}{lr}\text { Diminuição do } \\
\text { número } & \text { de } \\
\text { encaminhamento } & \\
\text { para } & \text { o } \\
\text { cardiologista. } & \end{array}$ & Dr. Raynner & $\begin{array}{l}\text { Atividade } \\
\text { contínua }\end{array}$ & 2019 \\
\hline $\begin{array}{lr}\text { Qualificação } & \text { de } \\
\text { toda a equipe da } \\
\text { ESF, para } \\
\text { melhorar } \\
\text { atendimento } \\
\text { desses pacientes. } \\
\end{array}$ & $\begin{array}{lr}\text { Conhecer } & \\
\text { exatamente } & \text { a } \\
\text { epidemiologia } & \text { da } \\
\text { HAS } & \text { na } \\
\text { comunidade. } & \end{array}$ & Dr. Raynner & $\begin{array}{l}\text { Atividade } \\
\text { contínua }\end{array}$ & 2019 \\
\hline
\end{tabular}

Fonte: Elaboração própria de acordo com dados coletados 
Quadro 8 - Plano de ações beta e suas metas

\begin{tabular}{|c|c|c|c|c|}
\hline $\begin{array}{l}\text { Ações do tipo } \\
\text { beta }\end{array}$ & Meta de Produto & Responsável & Prazo & $\begin{array}{l}\text { Início- } \\
\text { Fim }\end{array}$ \\
\hline $\begin{array}{lr}\text { Implementar } & \text { uma } \\
\text { farmácia } & \text { na } \\
\text { unidade. } & \end{array}$ & $\begin{array}{l}\text { Aumentar a adesão } \\
\text { do paciente ao } \\
\text { tratamento. }\end{array}$ & Enfermeira & $\begin{array}{l}\text { Atividade } \\
\text { contínua }\end{array}$ & 2019 \\
\hline $\begin{array}{l}\text { Coletar o material } \\
\text { para exame na } \\
\text { unidade }\end{array}$ & $\begin{array}{lr}\text { Aumentar } & \text { o } \\
\text { diagnóstico } & \text { de } \\
\text { hipertensão } & \\
\text { secundária. } & \end{array}$ & Enfermeira & $\begin{array}{l}\text { Atividade } \\
\text { contínua }\end{array}$ & 2019 \\
\hline $\begin{array}{l}\text { Planilha dos } \\
\text { pacientes com } \\
\text { HAS ou DM, com } \\
\text { a taxa de função } \\
\text { renal calculada. }\end{array}$ & $\begin{array}{l}\text { Conhecimento de } \\
\text { todos os pacientes } \\
\text { com HAS ou DM. }\end{array}$ & Dr. Raynner & $\begin{array}{l}\text { Atividade } \\
\text { contínua }\end{array}$ & 2019 \\
\hline
\end{tabular}

Fonte: Elaboração própria de acordo com dados coletados

Quadro 9 - Plano de ações gama e suas metas

Objetivo: Diagnosticar precocemente a hipertensão arterial sistêmica nas Unidades básicas de saúde.

Meta de resultado: Diminuição das complicações da HAS

\begin{tabular}{|l|l|l|l|l|}
\hline $\begin{array}{l}\text { Ações do tipo } \\
\text { gama }\end{array}$ & Meta de Produto & Responsável & Prazo & $\begin{array}{l}\text { Início- } \\
\text { Fim }\end{array}$ \\
\hline $\begin{array}{l}\text { Lista de pacientes } \\
\text { com HAS e/ou } \\
\text { DM, e dados } \\
\text { como obesidade, } \\
\text { circunferência } \\
\text { abdominal, última } \\
\text { consulta etc. }\end{array}$ & $\begin{array}{l}\text { Promoção } \\
\text { saúde; }\end{array}$ & $\begin{array}{l}\text { Atividade } \\
\text { contínua }\end{array}$ & 2019 \\
\hline $\begin{array}{l}\text { Solicitação de } \\
\text { exames anuais do } \\
\text { público com fator } \\
\text { de risco. }\end{array}$ & $\begin{array}{l}\text { Prevenção } \\
\text { secundária para } \\
\text { HAS, tratando } \\
\text { segundo diretrizes } \\
\text { da sociedade } \\
\text { brasileira de } \\
\text { cardiologia; sequelas }\end{array}$ & Dr. Raynner & $\begin{array}{l}\text { Atividade } \\
\text { contínua }\end{array}$ & 2019 \\
\hline $\begin{array}{l}\text { Consulta médica } \\
\text { anual e palestra } \\
\text { de prevenção de } \\
\text { HAS semestrais. }\end{array}$ & $\begin{array}{l}\text { Evitar ardiara } \\
\text { devido }\end{array}$ & $\begin{array}{l}\text { Atividade } \\
\text { contínua }\end{array}$ & 2019 \\
\hline
\end{tabular}

Fonte: Elaboração própria de acordo com dados coletados 
Como mostrado acima, sendo o comprometimento muito importante para haver menor rejeição às mudanças foi observado que 55,9\% dos servidores apresentam envolvimento moderado e baixo com suas instituições, $17,8 \%$ têm baixo comprometimento e $44,0 \%$ dos servidores possuem alto comprometimento. Nesse mesmo estudo citado anteriormente foi visto que o fator mais relevante para resistência foi a indecisão e inconclusão com o processo de mudança ${ }^{35}$.

Apesar dessa resistência e seus fatores causais, frequentemente presente, há que ter mais empenho para tentar melhor a qualidade no atendimento a HAS, pois só no ano de 2006 foram internadas no Brasil 146.426 pessoas por causas hipertensivas sensíveis a atuação da atenção primária. Se juntássemos todas as internações por condições sensíveis à atenção básica deixariam de existir mais de 2,7 milhões de internações, representando cerca de $24 \%$ das internações do ano de $2006^{36}$.

Além disso, foram levantados os determinantes para a dificuldade do controle da hipertensão arterial e pode ser observado, que uma grande parte está relacionada a fatores culturais da população, como já foi bastante discutido em diversas fontes na literatura $^{37,38}$

\section{CONSIDERAÇÕES FINAIS}

A HAS foi detectada como principal agravo encontrado na população da UBS 15 de Ceilândia. É uma doença de controle ambulatorial que demanda acompanhamento periódico pela equipe de saúde, adesão ao tratamento medicamentoso e alteração do estilo de vida. O diagnóstico preciso seguido de orientações corretas evita a evolução da doença para quadros mais graves, de maior complexidade que demandam serviços de saúde especializados e de alto custo.

A identificação racionalizada dos problemas encontrados na unidade seguida do planejamento de ações com metas bem definidas permite melhorar a dinâmica de 
trabalho e obter maior eficiência do serviço. Com o planejamento é possível identificar as ações prioritárias a serem desenvolvidas na unidade (melhorar a capacitação e comprometimento da equipe, por exemplo) e as que demandam ações externas (continuidade de fornecimento de medicamentos, insumos e exames complementares).

Como exemplificado em todo o trabalho, após um estudo muito cuidadoso e extremamente individualizado para a unidade, chegaram-se a algumas ações. A maioria das ações, como pode se perceber, é relacionada principalmente à dinâmica de equipe que inicialmente se encontrava falha. As alterações de serviço são, sem dúvida, uma das maiores dificuldades a serem vencidas.

O objetivo elencado para esse trabalho foi cumprido em sua íntegra, sendo realizada uma boa discussão sobre os principais fatores que dificultam o controle da pressão arterial na unidade de saúde estudada. Foram traçados diversos planos de ação para eliminar as dificuldades. Há necessidade também de um novo estudo após a execução das ações previstas nesse projeto para avaliar sua eficácia.

Logo, a resolubilidade do problema lançado passa prioritariamente por uma melhor gestão de pessoas, insumos e infraestrutura, sendo esses as principais dificuldades encontradas para sanar a maioria das moléstias/problemas que as unidades do Brasil a fora se deparam.

\section{REFERENCIAS}

1- Coelho, I.B. As propostas de modelos alternativos em saúde. Disponível em: <http://www.nescon.medicina.ufmg.br/biblioteca/imagem/2147.pdf >. Acesso em: 8 ago. 2019.

2- Conselho Nacional de Secretários de Saúde: Planificação da atenção à saúde: um instrumento de gestão e organização da atenção primária e da atenção ambulatorial especializada nas redes de atenção à saúde. Brasília, CONASS, 2018. 300p 
3- Mendes, E.V. As redes de atenção à saúde. Brasília: Organização PanAmericana da Saúde, 2011.

4- Brasil. Ministério da Saúde. Portaria nº 2.436 de 21 de setembro de 2017. Brasília: Diário Oficial da República Federativa do Brasil, 2017.

5- Santos, C.M.L.; Ferreira, S.M.S.P.; Almeida, C.S. et al. Planejamento estratégico na promoção à saúde do idoso: uma experiência integradora academiaserviço-comunidade. Rev Inter Educ Saúde, Salvador, 2019 Outubro; 3(1): Doi: 10.17267/2594-7907ijhe.v3i1.2139 | ISSN 2594-7907

6- Reichert, A.P. da S.; Leônico, A.B. de A. Orientação familiar e comunitária na Atenção Primária à Saúde da criança. Ciência \& Saúde Coletiva, v. 1, p. 119-127, 2016.

7- Dorneles, T.C.; Oliveira, I.C.; Weiller, T.H. et al. O residente multiprofissional na construção da planificação da atenção primária à saúde: relato de experiência. Tempus, actas de saúde colet, Brasília, 10(4), 319-329, dez, 2016.

8- Costa, V.F.C. O Processo de implementação da tutoria da planificação da atenção primária em uma unidade laboratório de um município do interior de Goiás. Rev Cient Esc Estadual Saúde Pública Goiás "Cândido Santiago".2019;4(3):208215.

9- Rivera, F.J.U.; Artmann, E. Esquema Operativo do Planejamento Estratégico para o nível local. Revista do DAPS/ENSP, 1993

10-Arantes, L.J.; Shimizu, H.E.; Merchan-Hamann, E. Contribuições e desafios da Estratégia Saúde da Família na atenção primária à saúde no Brasil: revisão da literatura. Ciênc. saúde coletiva. 2016;21(5):1499-510. doi: 10.1590/141381232015215.19602015 
11-World Health Organization. 2008-2013 Action Plan for the Global Strategy for the Prevention and Control of Noncommunicable Diseases. WHO Global Report, 2005.

12-Brasil, Ministério da Saúde. Banco de dados do Sistema Único de Saúde DATASUS. Informações de Saúde, Sistema de Informações sobre Mortalidade. Disponível em http://www.datasus.gov.br/catalogo/sim.htm [Acessado em 28 de maio de 2019].

13-Vigitel Brasil 2018: vigilância de fatores de risco e proteção para doenças crônicas por inquérito telefônico: estimativas sobre frequência e distribuição sociodemográfica de fatores de risco e proteção para doenças crônicas nas capitais dos 26 estados brasileiros e no Distrito Federal em 2018 / Ministério da Saúde, Secretaria de Vigilância em Saúde, Departamento de Análise em Saúde e Vigilância de Doenças não Transmissíveis. - Brasília: Ministério da Saúde, 2019.132.:il. Disponível

em http://bvsms.saude.gov.br/bvs/publicacoes/vigitel_brasil_2018_vigilancia_fatores_ri $\underline{\text { sco.pdf }}$

14-Rolim, E.C. et al. UBS Itapoã DF eq. 7 - preparo para a planificação na UBS Itapoã: diagnóstico de território e ajustes de planos terapêuticos para hipertensão e diabetes em população de baixa renda de cidade satélite do DF. Braz. J. of Develop., Curitiba, v. 5, n. 7, p. 8426-8435 jul. 2019 ISSN 2525-8761

15-Artmann, E. O Planejamento Estratégico Situacional: A Trilogia Matusiana e uma Proposta para o Nível Local de Saúde (Uma Abordagem Comunicativa). Dissertação de Mestrado, Rio de Janeiro: Escola Nacional de Saúde Pública, Fundação Oswaldo Cruz, 1993 
16-Artmann, E.; Azevedo, C.S.; Sá, M.C. Possibilidades de aplicação do enfoque estratégico de planejamento no nível local de saúde: análise comparada de duas experiências. Rio de Janeiro: Cadernos de Saúde Pública, 13(4):723-740, out-dez, 1997.

17-Andrade, S.S.A; Stopa, S.R.; Brito, A.S. et al. Prevalência de hipertensão arterial autorreferida na população brasileira: análise da Pesquisa Nacional de Saúde, 2013. Epidemiol Serv Saúde. 2015;24(2):297-304.

18-Malta, D.C.; Stopa, S.R.; Szwarcwald, C.L.; Gomes, N.L. et al. Vigilância e o monitoramento das principais doenças crônicas não transmissíveis no Brasil: Pesquisa Nacional de Saúde, 2013. Rev Bras Epidemiol. 2015 dez;18(Supl 2):316. http://dx.doi.org/10.1590/1980-5497201500060002 . PMid:27008599.

19-Fontbonne, A. et al. Risk factor control in hypertensive and diabetic subjects attended by the Family Health Strategy in the State of Pernambuco, Brazil: the SERVIDIAH study. Cad Saúde Pública. 2013 jun;29(6):1195204. http://dx.doi.org/10.1590/S0102-311X2013000600016 . PMid:23778551.

20-Anchala, R.; Kannuri, N.K; Pant, H. et al. Hypertension in India: a systematic review and meta-analysis of prevalence, awareness, and control of hypertension. $\mathbf{J}$ Hypertens. 2014; 32:1170-1177. doi: 10.1097/HJH.0000000000000146

21-Filippi, A.; Sangiorgi, D.; Buda, S. et al. Study Group (2013). How many hypertensive patients can be controlled in "real life": an improvement strategy in primary care. BMC family practice, 14, 192. doi:10.1186/1471-2296-14-192

22-Schmidt, M.I. et al. Doenças crônicas não transmissíveis no Brasil: carga e desafios atuais. Lancet 2011; 61-74. 
23-Brasil. Ministério da Saúde. Plano de ações estratégicas para o enfrentamento das doenças crônicas não transmissíveis (DCNT) no Brasil, 2011-2022. Brasília: Ministério da Saúde; 2011.

24- World Health Organization. Global status report on noncommunicable diseases 2010. Geneva: WHO; 2011.

25-Dib, M.W.; Riera, R.; Ferraz, M.B. Estimated annual cost of arterial hypertension treatment in Brazil. Rev Panam Salud Publica. 2010 fev; 27(2):12531. http://dx.doi.org/10.1590/S1020-49892010000200006 . PMid:20339616.

26- Abegunde, D.O.; Mathers, C.D.; Adam, T. et al. The burden and costs of chronic diseases in low-income and middle-income countries. Lancet 2007; 370: 1929-38.

27-Furberg, C.D.; Sealey, J.E.; Blumenfeld, J.D. Unsuccessfully Treated Hypertension: A Major Public Health Problem With a Potential Solution, American Journal of Hypertension, Volume 30, Issue 9, September 2017, Pages 857860, https://doi.org/10.1093/ajh/hpx068

28-Malachias, M.V.B. et al. 7a Diretriz Brasileira de Hipertensão Arterial. Arq Bras Cardiol 2016; 107(3Supl.3):1-83

29-Williams, B. et al. 2018 ESC/ESH Guidelines for the management of arterial hypertension. European Heart Journal, Volume 39, Issue 33, 01 September 2018, Pages 3021-3104, https://doi.org/10.1093/eurheartj/ehy339

$\begin{array}{llll}\text { 30-Whelton, } & \text { P.K. } & \text { et } & \text { al. }\end{array}$ ACC/AHA/AAPA/ABC/ACPM/AGS/APhA/ASH/ASPC/NMA/PCNA Guideline for the Prevention, Detection, Evaluation, and Management of High Blood Pressure in Adults. Hypertension. 2018;71:e13-e115. DOI: 10.1161/HYP.0000000000000065. 
31-Elias, S. M. Employees commitment in times of change: assessing the importance of attitudes towards organizational change. Journal of Management, 35(1), 2009, 37-55. doi: 10.1177/0149206307308910

32-Peccei, R.; Giangreco, A.; \& Sebastiano, A. The role of organizational commitment in the analysis of resistance do change: co-predictor and moderator effects. Personnel Review, 40(2), 185-204, 2011. doi: 10.1108/00483481111106075 33-Coghlan, D. A person-centred approach to dealing with resistance to change. Leadership and Organization Development Journal, 14(4), 10-14, 1993. doi: $10.1108 / 01437739310039433$

34-Lambert, G. E., \& Hogan, L. N. Wanting change: the relationship of perceptions of organizational innovation with correctional staff job stress, job satisfaction, and organizational commitment. Criminal Justice Police Review, 21(2), 160-184, 2010. doi: $10.1177 / 0887403409353166$

35-Marques, A.L. et al. Relações entre resistência a mudança e comprometimento organizacional em servidores públicos de Minas Gerais. Rev. adm. contemp.,Curitiba , v. 18, n. 2, p. 161-175, Apr. 2014.

36- Alfradique, M.E. et al. Internações por condições sensíveis à atenção primária: a construção da lista brasileira como ferramenta para medir o desempenho do sistema de saúde (Projeto ICSAP - Brasil). Cad Saúde Pública. 2009 jun;25(6):133749. http://dx.doi.org/10.1590/S0102-311X2009000600016 . PMid:19503964.

37-Giovanella, L.; Mendonça, M.H.M. de. Atenção Primária à Saúde. In: FUNDAÇÃO OSVALDO CRUZ (Fiocruz) (Ed.). Políticas e sistemas de Saúde no Brasil. Rio de Janeiro: [s.n.], [s.d.]. p. 575-626.

38-Fernandez, J.C.A. Determinantes culturais da saúde: uma abordagem para a promoção de equidade. Saúde soc., São Paulo, v. 23, n. 1, p. 167-179, mar. 2014. 